Ferrata Storti Foundation

\title{
Cryptic non-canonical splice site activation is part of the mechanism that abolishes multimer organization in the c.2269_2270del von Willebrand factor
}

\author{
Viviana Daidone, ${ }^{1}$ Eva Galletta, ${ }^{1}$ Luigi De Marco $^{2}$ and Alessandra Casonato ${ }^{1}$
}

${ }^{1}$ University of Padua Medical School, Department of Medicine, First Chair of Internal Medicine, Padua and ${ }^{2}$ IRCCS, C.R.O. Aviano, Department of Translational Research, Stem Cells Unit, Aviano, Italy

Haematologica 2020

Volume 105(4):1120-1128

\section{Correspondence: \\ ALESSANDRA CASONATO \\ sandra.casonato@unipd.it \\ Received: April 2, 2019. \\ Accepted: July 10, 2019. \\ Pre-published: July 18, 2019.}

doi:10.3324/haematol.2019.222679

Check the online version for the most updated information on this article, online supplements, and information on authorship \& disclosures: www.haematologica.org/content/105/4/1120

\section{(C)2020 Ferrata Storti Foundation}

Material published in Haematologica is covered by copyright. All rights are reserved to the Ferrata Storti Foundation. Use of published material is allowed under the following terms and conditions:

https://creativecommons.org/licenses/by-nc/4.0/legalcode. Copies of published material are allowed for personal or internal use. Sharing published material for non-commercial purposes is subject to the following conditions:

https://creativecommons.org/licenses/by-nc/4.0/legalcode, sect. 3. Reproducing and sharing published material for commercial purposes is not allowed without permission in writing from the publisher.

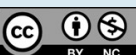

\section{ABSTRACT}

W Te report a new pathogenic mechanism in von Willebrand disease involving the use of a non-canonical splicing site. The proband, carrying the homozygous c.2269_2270del mutation previously classified as a type 3 mutation, showed severely reduced plasma and platelet von Willebrand factor antigen levels and functions, and no factor VIII binding capacity. A particular von Willebrand factor multimer pattern emerged in plasma, characterized by the presence of only two oligomers: the dimer and an unusually large band, with no intermediate components. There were von Willebrand factor multimers in platelets, but each band ran more slowly than the normal counterpart. No anti-von Willebrand factor antibodies were detectable. The proband was classified as having severe type 1 von Willebrand disease. Seeking the relationship between phenotype and genotype, we found the c.2269_2270del mutation associated with three different RNA: r.2269_2270del (RNAI), giving a truncated von Willebrand factor protein; r.[2269_2270del;2282_2288del] (RNAII), resulting from activation of a cryptic "AG" splicing site; and r.[2269_2270del;2281_2282insAG] (RNAIII), where the wild-type "AG" acceptor of exon 18 was retained due to the non-canonical 2279-2280 "CG" acceptor splicing site being used. The aberrant RNAII and RNAIII caused the alteration of the furin cleavage and binding sites, respectively, both resulting in a von Willebrand factor protein characterized by the persistence of von Willebrand factor propeptide, as confirmed by western blot analysis of the recombinant mutated von Willebrand factor molecules produced in vitro. Taken together, these findings explain the residual von Willebrand factor synthesis, slower-running multimers, and absent factor VIII binding capacity. The apparently pure gene null mutation c.2269_2270del profoundly alters von Willebrand factor gene splicing, inducing a complex RNA expression pattern.

\section{Introduction}

Von Willebrand factor (vWF) is a multimeric glycoprotein involved in the early stages of the hemostatic process. Synthesized by endothelial cells and megakaryocytes, vWF has two main functions: (i) it guarantees platelet recruitment and aggregation at the site of vascular injury; and (ii) it transports factor VIII (FVIII) in the blood circulation. ${ }^{1.3} \mathrm{vWF}$ function depends on its proper multimeric organization, comprising a set of discrete oligomers ranging from 500,000 to more than 20 million Daltons in size, the larger forms having the greater hemostatic capacity. ${ }^{4} \mathrm{vWF}$ is synthesized as a monomer of 2,813 amino acids, including a signal peptide of 22 amino acids, a propeptide of 741 amino acids, and a mature molecule of 2,050 amino acids. After dimerization in the endoplasmic reticulum (ER), vWF is multimerized in the Golgi apparatus by an inter-chain disulfide bond forming between cysteine residues 1099 and 1142 of two vWF dimers, thanks to the contribution of vWF propeptide (vWFpp). The propeptide is then cleaved by furin, and the mature vWF molecule is either 
stored (in $\alpha$-granules in platelets, or in Weibel-Palade bodies in endothelial cells) or secreted immediately in the circulation. ${ }^{5-7}$ After its secretion, vWF is recognized by the proteolytic enzyme ADAMTS13 (a disintegrin and metalloproteinase with a thrombospondin type 1 motif, member 13), which promptly cuts the multimeric molecule, giving rise to the oligomer pattern characteristic of $\mathrm{VWF}^{8-10}$ Reduced levels of vWF, or abnormalities in vWF, are associated with von Willebrand disease (vWD), the most common inherited hemorrhagic disorder. vWD is classified as type 1 (a partial quantitative vWF defect, with a normal or almost normal multimer organization and function), type 3 (total absence of vWF), or type 2 (which includes various structural/functional vWF abnormalities and is further classified as types $2 \mathrm{~A}, 2 \mathrm{~B}, 2 \mathrm{M}$ or $2 \mathrm{~N}$, depending on which vWF domain is impaired). ${ }^{11}$ VWD is caused by mutations in the vWF gene, ${ }^{12}$ but many other genetic and environmental factors have a role in modulating circulating vWF levels, including $\mathrm{ABO}$ blood group, age, infections, stress, and hormone therapy. ${ }^{13-}$ ${ }^{15}$ The increasing use of genetic analysis (vWF gene sequencing) for diagnostic purposes is improving our ability to characterize $\mathrm{vWD}$, especially in cases of type $1 \mathrm{vWD}$, which is the most difficult to diagnose correctly. Numerous mutations have emerged as causes of vWD, along with frequent cases of multiple vWF defects, and even more complex genetic pictures in which both quantitative and qualitative $v W F$ abnormalities co-exist. ${ }^{12,16} v W F$ cDNA sequencing showed that apparently silent mutations can activate cryptic splice sites or alter regulatory splicing elements, inducing exon skipping or RNA sequence deletions that often result in the formation of premature termination codons (PTC). ${ }^{17,18}$ These aberrant vWF mRNA can undergo nonsense-mediated mRNA decay (NMD), a mechanism that triggers the degradation of mutated transcripts bearing PTC to avoid the synthesis of truncated proteins potentially deleterious to the cell. ${ }^{19,20}$

The above-described processes all contribute to making vWD a very complex and heterogeneous disorder, and new cases often pose a real diagnostic challenge. Here we describe a very particular vWD phenotype that is due to an equally particular genetic condition. The causative c.2269_2270del mutation was found to abolish vWF production, acting through a complex mechanism that also includes the recognition of a cryptic non-canonical splicing site in the $v W F$ gene. To our knowledge, this is the first work to study the involvement of this rare, and still poorly understood, mechanism in the pathogenesis of vWD.

\section{Methods}

\section{Patients}

The patient investigated (proband) is a 50-year-old male with a lifelong history of severe bleeding. His 70-year-old mother and 76year-old father, and his offspring (an 18-year-old daughter and a 15 -year-old son) were also investigated. The subjects' bleeding scores (BS) were calculated with the International Society on Thrombosis and Haemostasis (ISTH) Bleeding Assessment Tool (BAT). ${ }^{21}$ All subjects gave written informed consent in accordance with the Declaration of Helsinki.

\section{Hemostatic analysis}

Basic hemostatic tests were performed as explained elsewhere. ${ }^{22-24}$ For more details, see also the Online Supplementary Methods. The proband's samples were diluted less than the control plasma to adjust for his markedly reduced vWF levels. vWF multimers were analyzed according to previously reported procedures. ${ }^{25}$ Desmopressin (DDAVP) was administered subcutaneously at a dose of $0.3 \mu \mathrm{g} / \mathrm{kg}^{26}$ Anti-vWF antibody detection was performed by ELISA. ${ }^{27}$ ADAMTS13 activity was ascertained using FRETS-vWF73 (Peptide International, Lexington, KY, USA) as a substrate. A negative test was defined as a titer $\leq 16 \mathrm{IU} / \mathrm{mL}$.

\section{Genetic analysis}

The patients' genomic DNA was obtained from whole blood; total RNA was extracted from platelets (see the Online Supplementary Methods for more details). Sequencing reactions were performed in an ABI3130 XL Genetic Analyzer.

\section{Digital droplet polymerase chain reaction}

The digital droplet polymerase chain reaction (ddPCR) assays were performed with the QX200 ddPCR system (Bio-Rad, Hercules, CA, USA) using the ddPCR ${ }^{\mathrm{TM}}$ Supermix for Probes (BioRad), with different primer pairs and probes specifically designed for aberrant mRNA sequences (Online Supplementary Methods). The assay was run in multiplex, starting from $25 \mathrm{ng}$ of the proband's cDNA, at $\mathrm{T}=56^{\circ} \mathrm{C}$ and adding $2 \% \mathrm{DMSO}$ to the PCR mix. cDNA was obtained by retrotranscribing RNA extracted from the proband's platelets, as explained above.

\section{In vitro expression experiments}

The $\mathrm{pRc} / \mathrm{CMV}$-vWF vector containing the wild-type (WT) human full-length vWF cDNA was mutated by site-directed mutagenesis using the QUIKCHANGE II XL kit (Stratagene, La Jolla, CA, USA). The primer sequences used for mutagenesis are listed in the Online Supplementary Methods. The vectors were used to transiently transfect Human Embryonic Kidney 293T (HEK293T) cells. Transfections were performed in T25 flasks using the Lipofectamine 2000 transfection reagent (Life Biotechnology, Carlsbad, CA, USA) and $4 \mu \mathrm{g}$ of vector/T25. After 48 hours of transfection, the media containing the recombinant vWF (rvWF) were collected, and the rvWF was quantified with ELISA. The rvWF intracellular content was also measured after lysing cells with a $2 \%$ Triton-X-100 solution, as previously reported. ${ }^{17}$ The results of each transfection experiment were calculated as the mean of three replicates.

\section{SDS-PAGE and western blot}

rvWF samples were run in reducing conditions using the XCell SureLock Mini electrophoresis system (Invitrogen, CA, USA) on a Novex Tris-Glycine 3-8\% precast gel (Invitrogen). After blotting, the membrane was incubated with a polyclonal rabbit anti-vWF (DAKO, Glostrup, Denmark), and then with a polyclonal anti-rabbit-HRP antibody (for further details see the Online Supplementary Methods).

\section{Results}

\section{Characterization of a complex von Willebrand disease phenotype}

The proband's main symptoms included epistaxis, oral cavity bleeding, frequent hematomas, and bleeding from minor wounds. He also reported episodes of hemarthrosis and severe hemorrhagic complications after tooth extractions. His BS was 30 (vs. 0-3 in normal males). His mother and father (who were consanguineous) were almost asymptomatic, and so were his offspring. Hemostatic tests on the proband revealed a severely impaired hemostatic picture, with a very prolonged activated partial thrombo- 
A

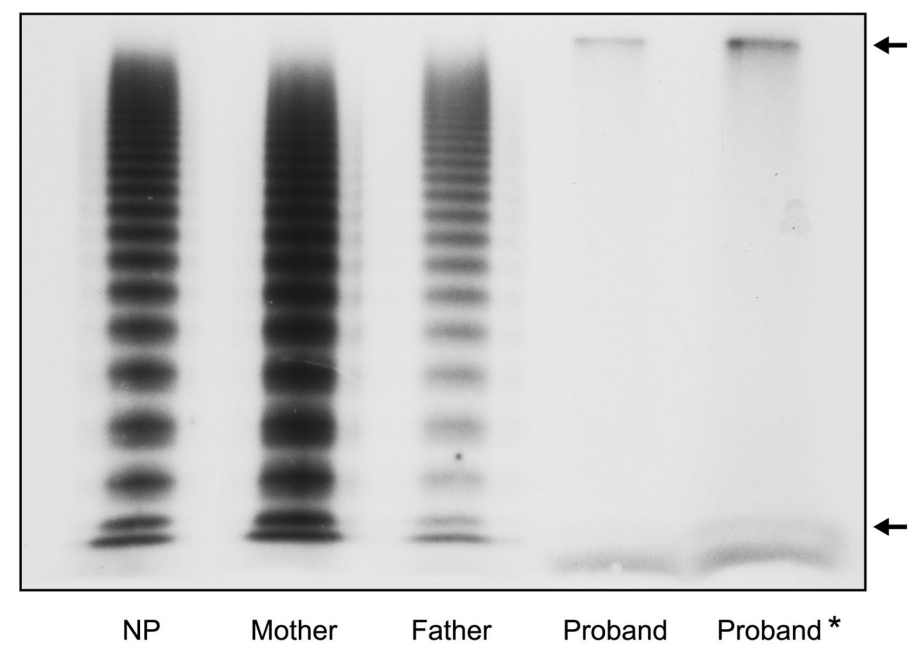

B

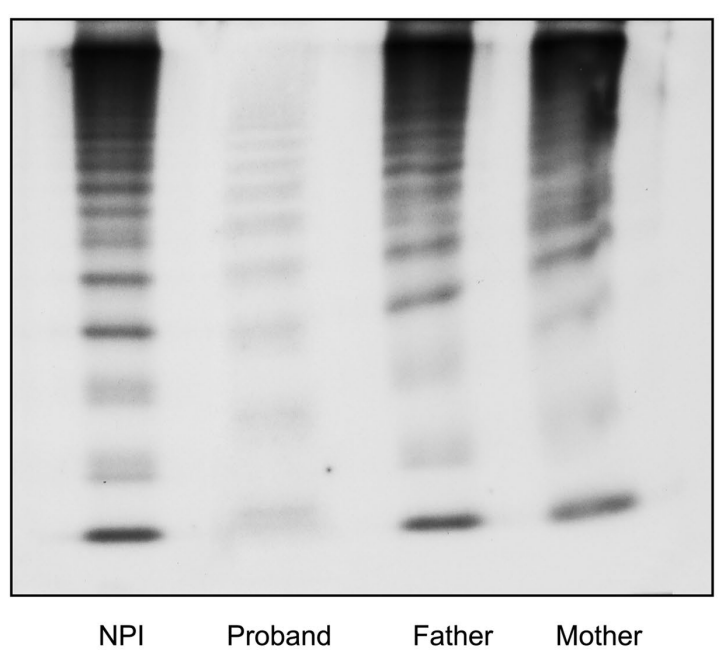

Figure 1. Plasma (A) and platelet (B) von Willebrand factor (vWF) multimer patterns in the proband and his parents, compared with normal plasma (NP) and platelets (NPI). Proband multimer pattern was obtained by plasma diluted $1: 5$ (left) and 1:2.5 (right*), as compared to the 1:20 dilution of the other samples. Large vWF multimers are at the top, smaller ones at the bottom. All vWF oligomers were absent except for the protomer and an ultra-large band in the patient's plasma, while both low-molecular-weight and intermediate oligomers are present (albeit significantly reduced) in his platelets. The patient's extra band below the first band of the other samples represents the non-specific binding of the anti-vWF polyclonal antibody to proteins other than vWF. This is more evident in the proband because of the less pronounced dilution of the samples. The slower running of each platelet vWF oligomer with respect to the normal counterpart provides evidence of the vWFpp persistence.

plastin time (aPTT), no PFA100 occlusion, and very low FVIII:C and plasma vWF:Ag levels (3.4 U/dL and 1.2 U/dL, respectively). Platelet $\mathrm{vWF}$ content was also severely reduced $(1.7 \mathrm{U} / \mathrm{dL})$, and plasma vWF was functionally impaired (vWF:RCo $3.8 \mathrm{U} / \mathrm{dL}$ and vWF:CB $1.9 \mathrm{U} / \mathrm{dL}$ ). vWF:FVIIIB was undetectable (Table 1), suggesting a defective FVIII carrier function. All hemostatic parameters were normal, or slightly reduced, in both the proband's parents and offspring (Table 1). Analyzing the proband's vWF multimer pattern revealed no circulating oligomers, apart from two bands: a lower one corresponding to the vWF dimer, and an unusually large band significantly higher than the highest vWF band seen in normal plasma (Figure 1A). On the other hand, the proband's platelet vWF contained low and intermediate oligomers, albeit in significantly reduced quantities, but no large vWF multimers. In addition, each oligomer was found to run less than the corresponding band in normal platelets, a finding resembling the picture seen when there is a persistence of vWFpp $^{28}$ (Figure 1B). DDAVP challenge prompted an approximately 8-fold maximum increase in plasma vWF:Ag (120 minutes after injection), associated with a stronger representation of the two previously-identified circulating vWF oligomers (the dimer and the ultralarge band), but no other low or intermediate vWF oligomers appeared (Figure 2). No anti-vWF antibodies were detected in the plasma. The proband's ADAMTS13 activity level was normal at $116 \%$ (normal range $65-130 \%$ ), and no anti-ADAMTS13 antibodies were detected.

\section{Patients' genetic characterization}

$v W F$ gene sequencing revealed that the proband was homozygous for the c.2269_2270del mutation in exon 17, and the same mutation was found in both his parents and offspring at heterozygous level. This genetic condition has been described in the literature as a gene null mutation predicted to generate a truncated vWF protein. ${ }^{29}$ It has been associated with type 3 VWD, but such a diagnosis is incompatible with our patient's phenotype. He had severely reduced but still detectable vWF levels, there were vWF oligomers in his platelets, and he also responded to DDAVP: this picture more closely resembles severe type 1 than type 3 VWD. The proband's total RNA was extracted and retrotranscribed, and his $v W F$ cDNA was analyzed to clarify this apparent genotype-phenotype contradiction, and the cause of his residual vWF synthesis.

The finding of a noncanonical splicing site being used in the $v W F$ gene - Sequencing of the proband's vWF cDNA confirmed the homozygous c.2269_2270del mutation, and revealed a second, unexpected sequence anomaly. Starting 


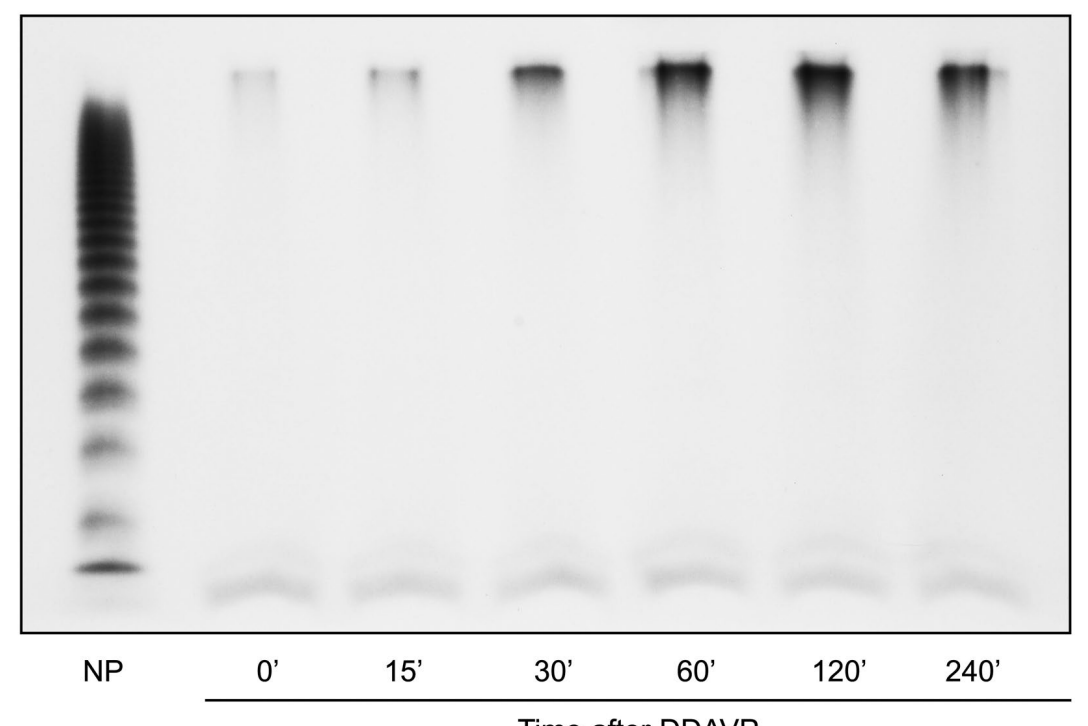

Time after DDAVP
Figure 2. The proband's vWF multimer pattern before $\left(0^{\prime}\right)$ and at various times after desmopressin (DDAVP) administration. The proband's multimer pattern was obtained from plasma diluted 1:2.5, instead of the 1:20 dilution used for the normal plasma (NP) Throughout the test, there was no sign of any discontinuous oligomers or additional discrete bands other than the protomer and the ultralarge band, while the two bands already seen before administering DDAVP appeared significantly increased. On the other hand, there was no evidence of any increase in the aspecific band below the dimer. from position c.2281, at the beginning of exon 18, the chromatogram became triple, a condition not found in the patient's genomic DNA, suggesting the co-existence of more than one mRNA species. To separate and characterize these sequences, the PCR-amplified cDNA fragment spanning exons 15-18 was cloned into a TA-vector, then sequenced again. Three vWF mRNA species were thus identified: (i) the r.2269_2270del (RNAI), lacking the CT dinucleotide at position 2269-2270; (ii) the r.[2269_2270del;2282_2288del] (RNAII), in which the first seven nucleotides of exon 18 were also deleted (position c.2282-2288); and (iii) the r.[2269_2270del;2281_2282insAG] (RNAIII), where an AG insertion was found 11 nucleotides downstream from the CT deletion (Figure 3). The fact that neither the c.2282_2288del nor the c.2281_2282insAG mutations had been found in the patient's genomic DNA indicates that they were the result of an altered splicing of exon 18. The deletion of 7 nucleotides in RNAII could be explained by the use of the AG nucleotides at position c.2287-2288 as the acceptor site, instead of the WT one. This hypothesis was confirmed by an in silico analysis with the Human Splicing Finder 3.1 software, which predicted the c.22872288 AG dinucleotide as a potential cryptic acceptor site, with a consensus of 72.4 (in a range from 1 to 100 , where 100 is the score corresponding to the normal splicing sites). On the other hand, the use of a non-canonical acceptor splicing site (the CG nucleotide pair at position c.2282-4_2282-3) might explain the retention of the c.2281-2282 AG dinucleotide in RNAIII, a pathogenic mechanism hitherto never described in vWD. The AG retention was confirmed by repeating the total RNA extraction and retrotranscription process twice, using different enzymes.

Genotype-phenotype correlation - Three different vWF mRNA were thus found associated with the c.2269_2270del genomic mutation. The RNAI form resulted in a truncated vWF (p.Leu757Valfs*22, indicated as P1 from now on), due to the generation of a premature translation stop codon as a consequence of the c.2269-2270 CT deletion. In RNAII, deriving from the usage of a cryptic
AG splicing site, the further 7 nucleotide deletion (c.22822288) restored the altered reading frame, producing a mature vWF slightly shorter than normal, and lacking the Arg763-Ser764 furin cleavage site (p.L757_R763delinsVSSO, named P2 from now on). In RNAIII, the c.2281-2282 AG retention resulting from the use of a non-canonical CG acceptor splicing site restored the reading frame lost due to the upstream CT dinucleotide deletion, producing an in-frame, full-length $v W F$, but with an altered 757-761 amino acid sequence (p.L757_761delinsVSSOG, named P3), corresponding to the consensus binding site of furin. ${ }^{30}$ Assuming no contribution of RNAI to vWF production (the P1 vWF cannot dimerize), the patient's phenotype should be due to the co-existence of two mutated vWF species (P2 and P3 $\mathrm{vWF}$ ), both resistant to the enzymatic action of furin. In our patient, the residual synthesis of mutated $v W F$, characterized by the persistence of its propeptide, confirms the presence of these P2 and P3 vWF forms.

Different expression of RNAI, RNAII and RNAIII - A multiplex ddPCR assay was performed on the proband's cDNA, to examine the levels of expression of the three mRNA. RNAI was found to be the dominant species (6.3 copies/ng, $83 \%$ of the total vWF mRNA), followed by RNAII (0.96 copies/ng, $12 \%$ of the total vWF mRNA), and RNAIII ( 0.37 copies $/ n g, 5 \%$ of the total vWF mRNA). That the RNAI species encoding for the truncated p.Leu757Valfs*22 $v W F$ accounts for more than $80 \%$ of the patient's $v W F$ mRNA is consistent with his severe quantitative $v W F$ defect.

In vitro expression experiments - To establish the effect of the single and combined aberrant mRNA on vWF synthesis and release, we ran in vitro experiments using HEK293T cells and vectors pRc/CMV-vWF, pRc/CMV-vWF-RNAI, pRc/CMV-vWF-RNAII and pRc/CMV-vWF-RNAIII, encoding for WT, P1, P2 and P3 vWF, respectively.

$R N A I$ - No rvWF was found in the conditioned medium of HEK293T cells transfected with the pRc/CMV-vWFRNAI, while co-transfection of both the WT and the mutated vector (mimicking a heterozygous condition) induced a drop in rvWF production of about $30 \%$ (Figure 
4A). Analyzing the intracellular content of the transfected cells revealed a similar picture, with rvWF totally absent in the homozygous condition, and 30\% lower than normal $\mathrm{vWF}$ in the heterozygous condition (Figure 4B). The vWF:CB/vWF:Ag and vWF:FVIIIB/vWF:Ag ratios (respectively indicative of the multimer organization of $\mathrm{VWF}$ and its capacity for binding FVIII) did not change when RNAI was co-expressed with the WT, which means that no functional alterations are associated with P1 vWF (Figure $4 \mathrm{~A})$. These results demonstrate that the P1 mutation behaves like a gene null mutation, inducing a pure quantitative vWF defect.

RNAII and RNAIII - Transfection of HEK293T cells with $\mathrm{pRc} / \mathrm{CMV}$-vWF-RNAII caused a reduction in rvWF secretion of about $40 \%$ or $60 \%$, respectively, if the vector was expressed with or without the WT counterpart (Figure $4 \mathrm{~A}$ ). Conversely, there was an increase in rvWF intracellular content in the transfected cells that was more pronounced in the homozygous condition (Figure 4B), a finding suggestive of a partial retention of the P2 rvWF. A similar picture was seen for pRc/CMV-vWF-RNAIII: its expression was associated with a drop in rvWF secretion in the conditioned medium (of about $40 \%$ and $65 \%$ for the heterozygous and homozygous conditions, respectively), and a corresponding increase in the intracellular rvWF content (Figure 4B). The vWF:CB ratios were higher than normal, for both P2 and P3 rvWF (Figure 4A); in the homozygous condition, the ratios were $158.1 \%$ and
$197.3 \%$, respectively, of the WT (taken to be 100\%), suggesting the presence of larger than normal vWF multimers. On the other hand, the vWF:FVIIIB ratios were lower when P2 and P3 were expressed at heterozygous level (i.e. $57.3 \%$ and $52.0 \%$ of the WT, respectively), and vWF:FVIIIB was completely undetectable when each mutation was expressed at homozygous level. The P2 and P3 vWF mutations therefore both cause a partial quantitative defect associated with a defective FVIII binding capacity of the mutated vWF

Multimer analysis on the rvWF in the conditioned media confirmed the above results: oligomers of unusually high molecular weight were apparent for both P2 and P3 rvWF, with an accumulation of vWF oligomers on the boundary between the stacking and running gel and a relative representation of vWF multimers shifted towards the high-molecular-weight forms (Figure 5). All mutated rvWF oligomers also showed a delayed migration pattern, compared with WT (Figure 5), like the one seen in the patient's platelet multimers. This picture points to the persistence of vWFpp, a condition associated with vWF having an impaired FVIII carrier capacity. ${ }^{28}$

Co-transfection with RNAI, RNAII and RNAIII - Co-transfecting HEK293T cells with equal amounts of all vectors resulted in a $60 \%$ reduction in secreted rvWF, with a corresponding approximately $25 \%$ increase in intracellular rvWF content. In the conditioned medium, the rvWF:CB ratio was higher than normal, while vWF:FVIIIB levels

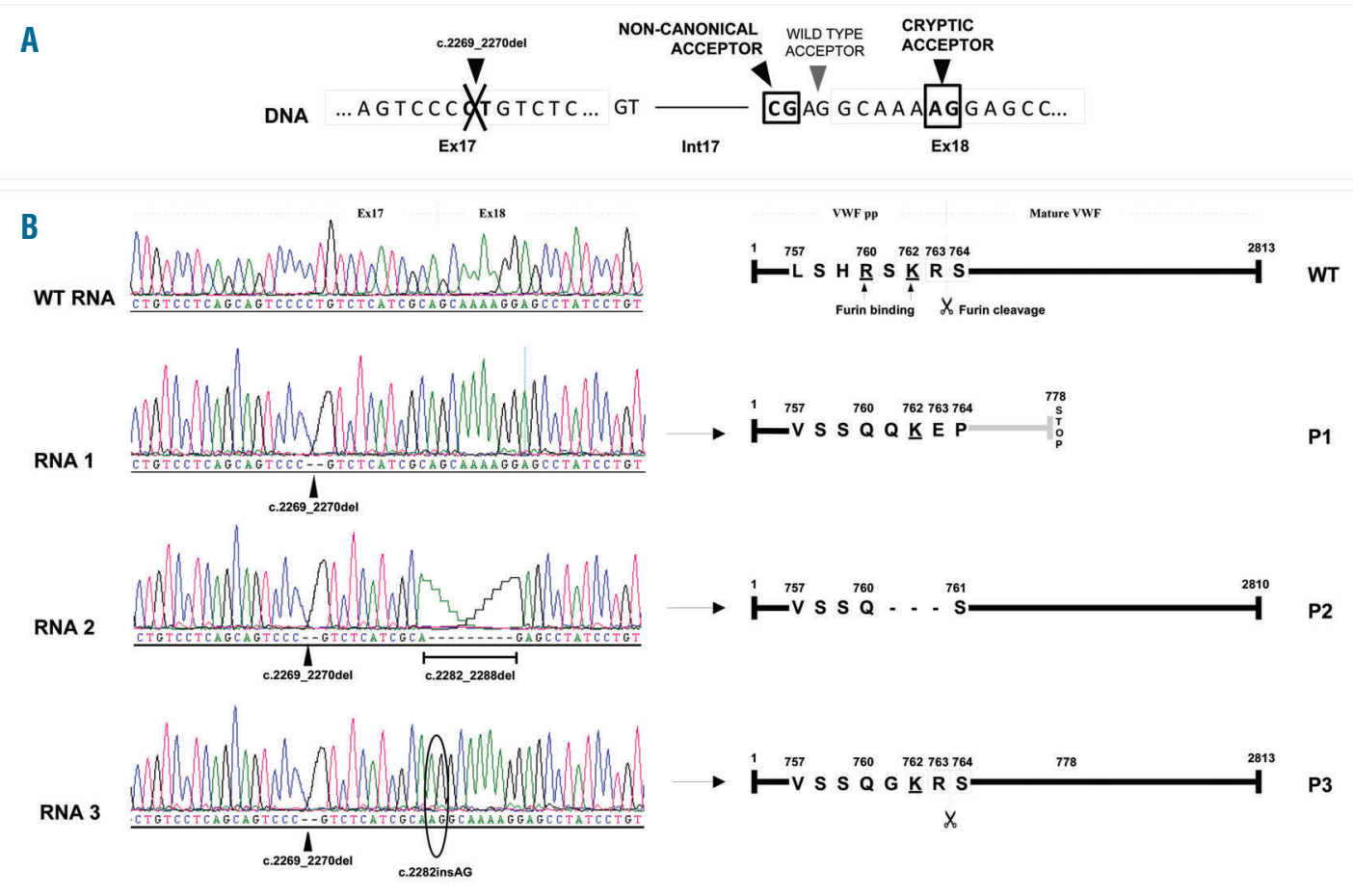

Figure 3. Schematic representation of the three mRNA associated with the c.2269_2270del von Willebrand factor (vWF) gene deletion, and the resulting mutated vWF proteins. RNAl deriving from the use of the wild-type (WT) donor and acceptor splicing sites encodes for a truncated vWF protein (P1, 778 amino acids long). RNAll resulting from the activation of a cryptic AG acceptor site within exon 18 encodes a protein with a 3 amino acids deletion, in which the furin cleavage site is lost (P2). Finally, RNAlll originates from the usage of a non-canonical CG acceptor site in intron 17, and results in a full-length vWF protein carrying a partially-altered furin recognition site (P3). vWFpp persistence is predicted for both P2 and P3 vWF, while no vWF secretion is expected for P1. The gray area identifies amino acids other than WT in P1, P2 and P3 vWF. Chromatograms were aligned with the SeqMan Pro software (DNASTAR, Madison, WI, USA). P1: p.Leu757Valfs 22 ; P2: p.L757_R763delinsVSSQ; P3: p.L757_761delinsVSSQG. 
were undetectable. rvWF multimer analysis revealed larger than normal oligomers, and a delayed oligomer migration (Figure 5).

SDS-PAGE and western blot analysis - Analyzing samples with SDS-PAGE and western blotting in reducing conditions revealed a protein band with a molecular weight corresponding to the unprocessed vWF-pp (about $340 \mathrm{KDa}$ ) in both P2 and P3 rvWF, while a band corresponding to the mature cleaved vWF $(250 \mathrm{KDa})$ was seen in the WT. These data confirmed the persistence of vWFpp in the P2 and P3 vWF (Figure 6).

\section{Discussion}

We report on a complex splicing alteration associated with the $v W F$ c.2269_2270del mutation that involves the use of a non-canonical splicing site in a case of severe type $1 \mathrm{vWD}$ characterized by a lack of multimer organization in the circulating vWF. The c.2269_2270del mutation was described in 2003 as a pure gene null mutation. ${ }^{29}$ Here, we demonstrate that it alters $v W F$ splicing, and induces the generation of three different aberrant mRNA species. The predominant specie, RNAI, encodes for the alreadyknown truncated p.Leu757Valfs*22 vWF (P1 in the text), associated with a pure quantitative vWF defect. ${ }^{29}$ The other two species found, RNAII and RNAIII, account for less than $20 \%$ of the total $v W F$ mRNA expression, and are produced by the activation of two cryptic acceptor splicing sites (a canonical "AG" and an unusual "CG") in exon 18 and intron 17 of the vWF gene, respectively. RNAII encodes for a mature vWF characterized by loss of the furin cleavage site (p.L757_R763delinsVSSQ, P2), while RNAIII has an altered furin recognition site that impairs its

Table 1. Main hemostatic and genetic findings in the proband and his parents.

\begin{tabular}{|c|c|c|c|c|c|c|c|c|c|c|c|}
\hline Patients & $\begin{array}{l}\text { aPTा } \\
\text { sec }\end{array}$ & $\begin{array}{c}\text { PFA100 } \\
\text { Sec }\end{array}$ & $\begin{array}{l}\text { RIPA } \\
\%\end{array}$ & $\begin{array}{l}\text { FVIII:C } \\
\text { U/dL }\end{array}$ & $\begin{array}{l}\text { WWFAg } \\
\text { U/dL }\end{array}$ & $\begin{array}{l}\text { WF:CB } \\
\text { U/dL. }\end{array}$ & $\begin{array}{l}\text { WWF:CB } \\
\text { ratio }\end{array}$ & $\begin{array}{l}\text { WWFFVIIIB } \\
\text { U/dL }\end{array}$ & $\begin{array}{l}\text { VWFFVIIIB } \\
\text { ratio }\end{array}$ & $\begin{array}{l}\text { Platelet WWF } \\
\text { U/dL }\end{array}$ & BS \\
\hline Proband & 54.6 & $>300$ & $0(5.6)^{*}$ & 3.4 & 1.2 & 1.9 & 1.58 & 0 & $\mathrm{NC}$ & 1.7 & 30 \\
\hline Mother & 22.8 & 160 & 72.9 & 209.6 & 114.3 & 104.9 & 0.91 & 118.0 & 1.03 & 97.6 & 1 \\
\hline Father & 31.0 & 198 & 74.2 & 89.1 & 39.3 & 40.4 & 1.03 & 38.2 & 0.97 & 103.0 & 0 \\
\hline Son & 97.9 & 150 & 65.4 & 119.6 & 66.0 & 61.4 & 0.93 & 61.1 & 0.97 & 74.4 & 1 \\
\hline Daughter & 93.2 & 145 & 72.3 & 108.2 & 56.3 & 63.6 & 1.13 & 55.0 & 0.97 & 83.9 & 1 \\
\hline Normal range & $24-36$ & $94-193$ & $72 \pm 12$ & $60-160$ & $60-160$ & $65-150$ & $\geq 0.75$ & $65-150$ & $\geq 0.74$ & $70-140$ & $0-3 / 0-5(\mathrm{M} / \mathrm{F})$ \\
\hline
\end{tabular}

aPTT: activated partial thromboplastin time; RIPA: ristocetin induced platelet aggregation at $1.2 \mathrm{mg} / \mathrm{mL}$ ristocetin concentration; *RIPA value at $1.5 \mathrm{mg} / \mathrm{mL}$ ristocetin concentration; BS: Bleeding Score, calculated by the ISTH Bleeding Assessment tool (BAT); M/F: male/female; NC: not calculable.

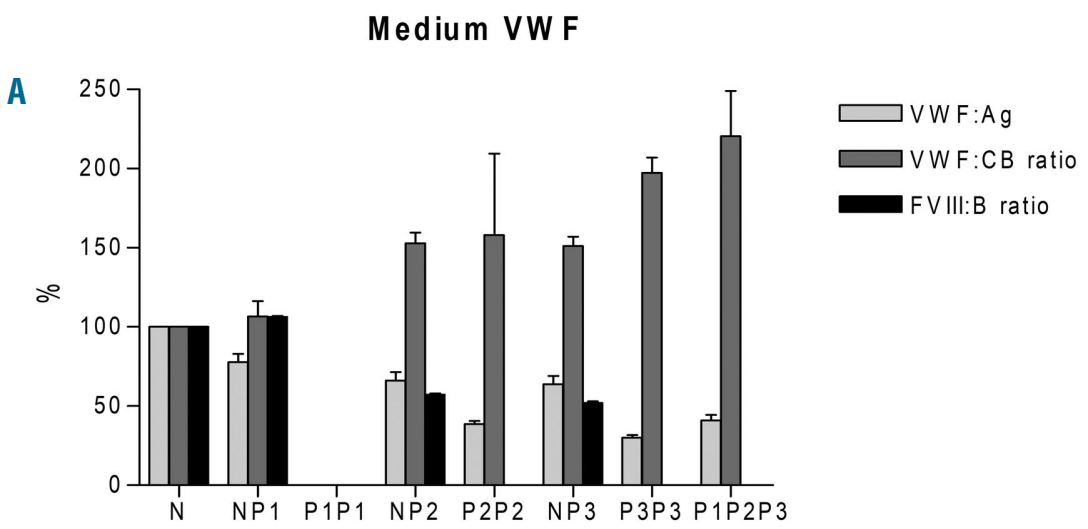

Intracellular VW F

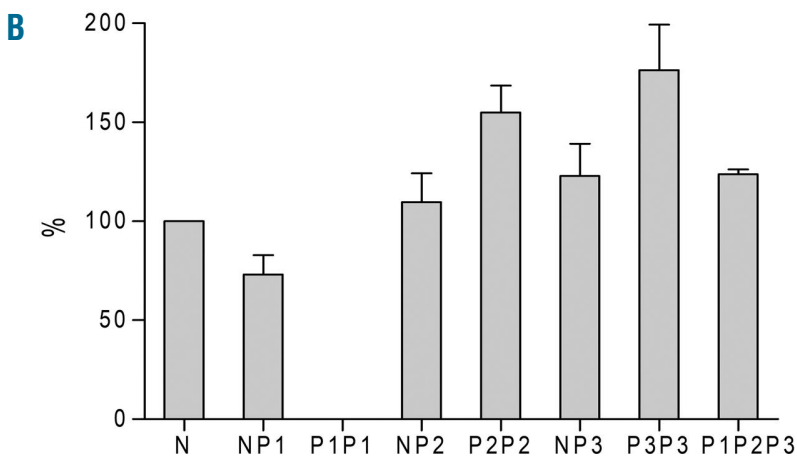

Figure 4. In vitro expression of the P1, P2, and P3 von Willebrand factor (vWF) mutations. Mean vWF:Ag, vWF:CB and vWF:FVIIIB ratios, as obtained in the conditioned culture media (A), and vWF:Ag in cell lysates (B) from HEK293T cells transfected with vectors containing the wild-type (WT) or the P1, P2, and P3 vWF. The gene null effect of the P1 mutation was demonstrated by the complete absence of recombinant vWF (rvWF), whereas both P2 and P3 induced the production of VWF protein. P1: p.Leu757Valfs*22; P2: p.L757_R763delinsVSSQ; P3: p.L757_761delinsVSSQG. 


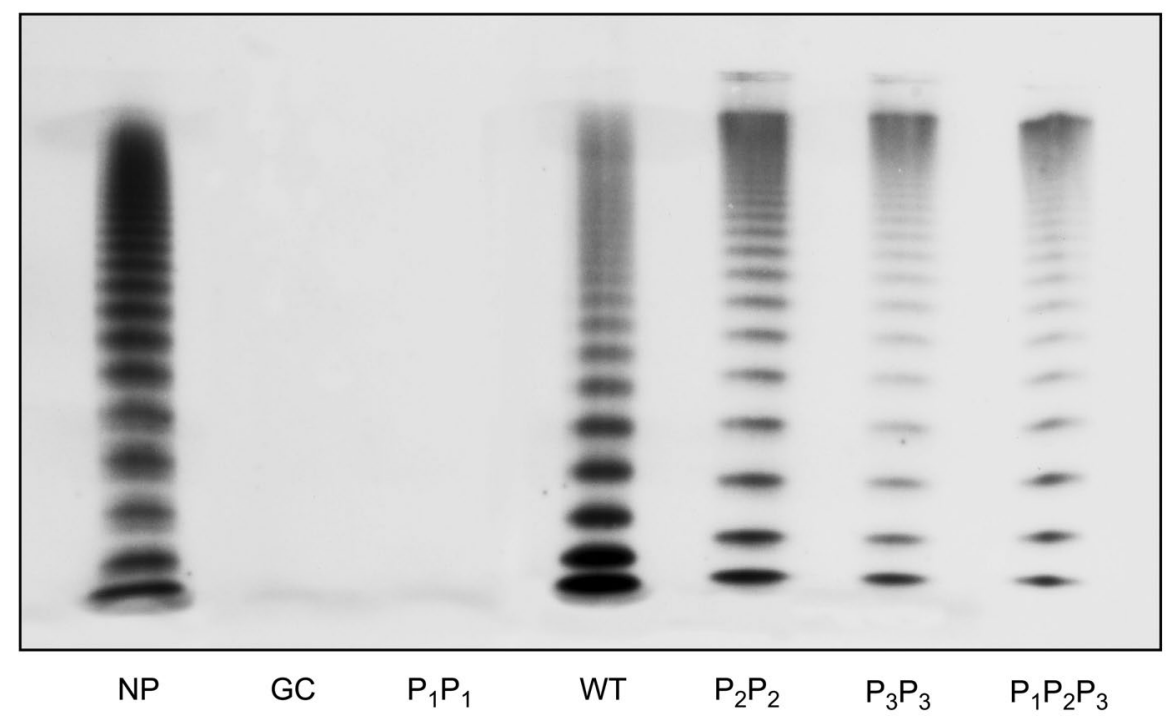

Figure 5. Multimer analysis of recombinant von Willebrand factor (rvWF). No rvWF was found in the conditioned medium from cells expressing the P1 vWF mutation. Both P2 and P3 rvWF were produced instead, and showed: (i) unusually large vWF multimers associated with the accumulation of multimers on the boundary of the running and stacking gel; (ii) an oligomer pattern that had shifted towards the high-molecular-weight forms; and (iii) oligomers with a slower migration pattern. P1: p.Leu757Valfs*22; P2: p.L757_R763delinsVSSQ; P3: p.L757_761delinsVSSQG.

enzymatic activity (p.L757_761delinsVSSQG, P3). ${ }^{30}$ Despite bearing a PTC, RNAI seems to escape the NMD control mechanism, as it accounts for more than $80 \%$ of total mRNA expression..$^{19,31}$

The proband was homozygous for the c.2269_2270del mutation, and carried no other mutations in his $v W F$ gene. He had a very complex and severe phenotype characterized by both quantitative and qualitative vWF alterations. Both plasma and platelet vWF levels were significantly decreased, leading to a diagnosis of severe type $1 \mathrm{vWD}$. The multimer organization of his circulating $v W F$ revealed a peculiar pattern involving the absence of any oligomers except for the vWF dimer, and an unusually ultra large band, without any intermediate components. This pattern did not change after DDAVP infusion, which increased the pre-DDAVP oligomers, without any other intermediate components being observed. On the other hand, the patient's platelet $v W F$ showed a multimer organization (albeit lacking in the large components), with each oligomer characterized by a delayed migration, a picture suggestive of a persistence of vWFpp. ${ }^{28}$ A similar vWF multimer pattern had previously been reported in a patient with systemic lupus erythematosus, who had the two above-described circulating vWF oligomers, but a normal platelet vWF content and multimer pattern in the presence of an anti-vWF antibody. ${ }^{26}$ In contrast, no antivWF antibodies were detectable in our patient.

The delayed migration of the proband's platelet vWF oligomers was attributable to the presence of the P2 and P3 vWF forms, both resistant to the action of furin, and consequently characterized by the persistence of the vWFpp. This was confirmed by our patient's undetectable vWF:FVIIIB values, as well as by the P2 and P3 rvWF forms, which point to his vWF having an impaired capacity to carry FVIII. ${ }^{32}$ The persistence of vWFpp is known to disrupt the capacity of vWF to bind FVIII because of a steric hindrance that makes the FVIII binding site in the D3 domain of vWF inaccessible. ${ }^{28}$
The DDAVP-induced release of VWF stored in the Weibel-Palade bodies, or a drop in ADAMTS13 concentration in the bloodstream are usually associated with the appearance of ultra large vWF multimers in the circulation. In both cases, all vWF oligomers are still present, however, whereas this was not so in our patient. There are also vWF mutations that interfere with the factor's recognition and/or binding by ADAMTS13, giving rise to the presence of unusually large vWF bands ${ }^{33,34}$ that are not normally organized, a picture not seen in our patient either. Indeed, the multimer pattern of the proband's circulating vWF appeared to be more similar to that of vWF inside normal endothelial cells. ${ }^{35}$ We know that the cysteine-linked vWF dimers grow in a concatemer via the formation of disulfide bonds at the N-terminal of the vWF molecule, with the contribution of vWFpp. After its secretion from endothelial cells, vWF remains anchored to the vessel wall and is then uncoiled by the action of the blood flow. This makes the Tyr1605-Met1606 bond accessible to ADAMTS13 for cleavage. The persistence of vWFpp could interfere with the unraveling of vWF during its release from endothelial cells; ${ }^{36-39}$ alternatively, the P2 and P3 vWF forms might be unable to remain anchored to the vessel wall during their release. Intriguingly, p-selectin protein (which is known to anchor vWF to the endothelial vessel wall under normal conditions) binds VWF in the D'D3 region, very near where our P2 and P3 mutations occur. ${ }^{40}$ While circulating vWF needs dynamic flow conditions for it to be cleaved by ADAMTS13 after its release, this might not be the case for vWF inside alpha-granules, and this would explain the different multimer organization in the two biological districts. A different contribution of aberrant vWF mRNA in endothelial cells and platelets is less plausible, but mRNA in endothelial cells would need to be explored to be able to verify this.

In vitro expression experiments confirmed that the c.2269_2270del mutation, hitherto considered a type 3 vWF defect, ${ }^{29,41}$ is actually associated with a residual syn- 


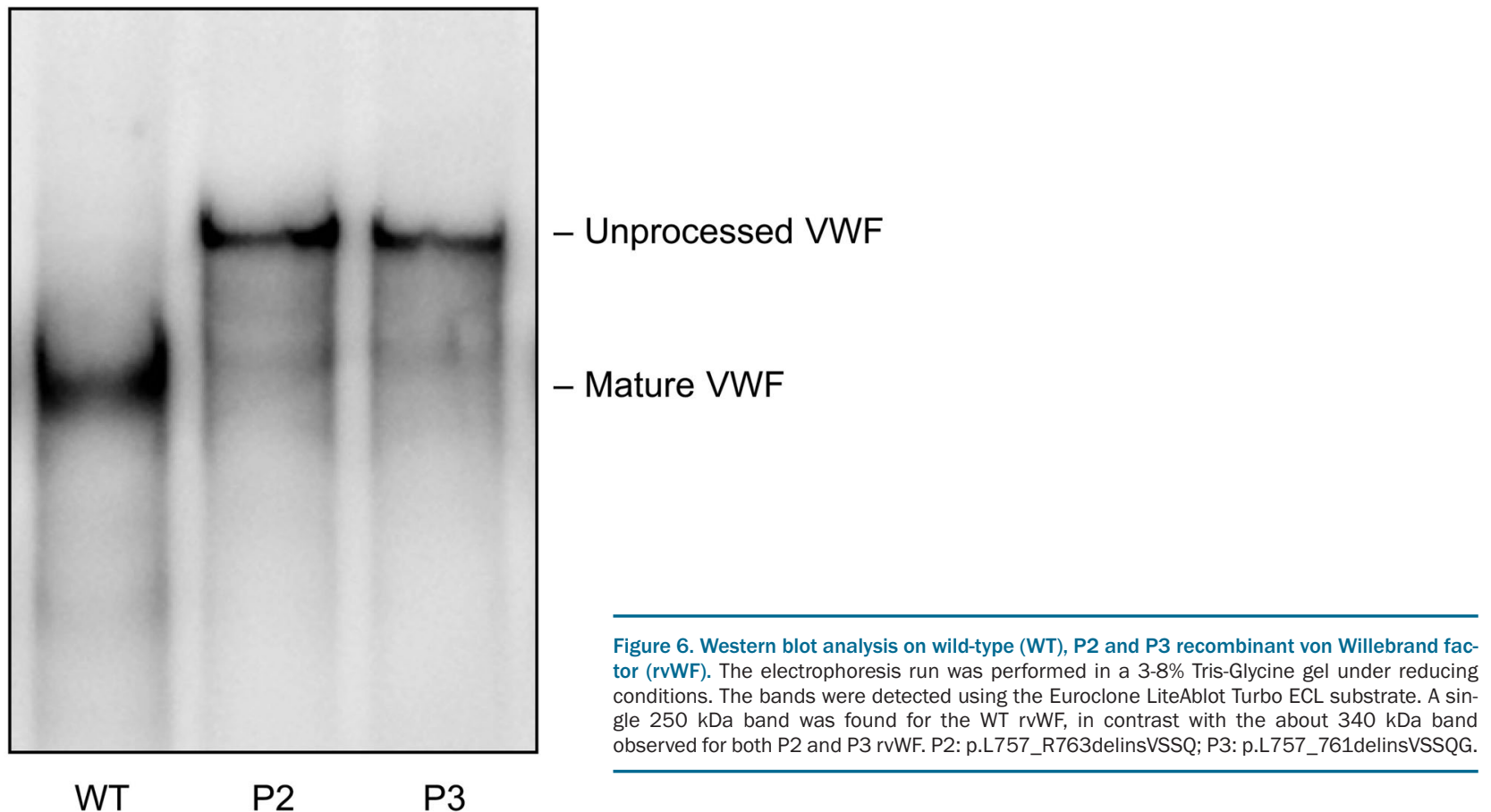

thesis of vWF due to the presence of RNAII and RNAIII forms. While RNAI (the prevalent $v W F$ mRNA form) produced no rvWF, both RNAII and RNAIII produced a rvWF molecule characterized by the persistence of vWFpp, as shown by the slower migration of each oligomer, and the weaker FVIII binding capacity. This finding was confirmed by western blot analysis, showing that both P2 and P3 rvWF were characterized by the presence of only the unprocessed vWF protein band. The presence of larger than normal vWF oligomers in the rvWF multimers, and their being in larger proportions than the low and intermediate forms, also confirm that the c.2269_2270del mutation induces ADAMTS13 resistance in vitro, and that vWF proteolysis can occur under static conditions (as seen also in the proband's platelets), thus explaining the proband's discrepant plasma and platelet vWF multimer patterns in vivo.

Interestingly, the c.2269_2270del mutation generates RNAII and RNAIII through the activation of two cryptic splicing sites in exon 18 and intron 17 of the vWF gene, respectively. The recognition of a cryptic splicing site is a known, not particularly rare, mechanism in $\mathrm{vWD}$, which can be responsible for vWF lacking altogether, ${ }^{42}$ or its levels only being reduced. ${ }^{43}$ In our patient, the two new acceptor sites activated due to the mutation are one a canonical "AG", and the other an unusual "CG" acceptor splicing site, respectively generating RNAII and RNAIII. A non-canonical splicing site has very rarely been identified in human genes (the sequences of 5' donor and 3' acceptor splicing sites almost always obey the GT-AG rule) ${ }^{44,45}$ and this is the first time it has been described in vWD. The new pathogenic mechanism behind VWD characterized in this work could have a significant impact on vWD diagnosis and management in future, bearing in mind the large proportion of vWD cases that, even nowadays, remain genetically unexplained.

To conclude, our investigation revealed the use of a noncanonical splicing site as a novel pathogenic mechanism behind vWD. This was associated with the previously reported, but only partially categorized, vWF c.2269_2270del mutation, and it resulted in a severe type $1 \mathrm{vWD}$ featuring an extremely particular vWF multimer organization, and lacking the ADAMTS13-induced oligomer pattern.

\section{Funding}

This work was supported by a grant from the MURST (ex $60 \%, 2016)$.

\section{References}

1. Sadler JE. Biochemistry and genetics of von Willebrand factor. Annu Rev Biochem. 1998;67:395-424.

2. Ruggeri ZM. Structure of von Willebrand factor and its function in platelet adhesion and thrombus formation. Best Pract Res Clin Haematol. 2001;14(2):257-279.

3. Fay PJ, Cumans JV, Walker FJ. von Willebrand factor mediates protection of FVIII from activated protein C-catalyzed inactivation. J Biol Chem. 1991;266(4): 2172-2177

4. Gralnick HR, Williams SB, Morisato DK. Effect of multimeric structure of the factor VIII/von Willebrand factor protein on binding to platelets. Blood. 1981;58(2):387-392.

5. Wagner DD, Marder VJ. Biosynthesis of von Willebrand protein by human endothelial cells: processing steps and their intracellular localization. J Biol Chem. 1984; 99(6):2123-2130.

6. Wagner DD, Lawrence SO, Ohlsson-
Wilhelm BM, Fay PJ, MarderVJ. Topology and order of formation of interchain disulfide bonds in von Willebrand factor. Blood 1987;69(1):27-32

7. Wagner DD, Fay PJ, Sporn LA, Sinha S, Lawrence SO, Marder VJ. Divergent fates of von Willebrand factor and its propolypeptide (von Willebrand antigen II) after secretion from endothelial cells. Proc Natl Acad Scie USA. 1987:84(7):1955-1959.

8. Sadler JE. von Willebrand factor assembly and secretion. Thromb Haemost. 2009;7 
Suppl 1:24-27.

9. Zheng X, Chung D, Takayama TK. Structure of von Willebrand factor-cleaving protease (ADAMTS13), a metalloprotease involved in thrombotic thrombocytopenic purpura. J Biol Chem. 2001;276(44):4105941063

10. Dent JA, Berkowitz SD, Ware J, Kasper CK, Ruggeri ZM. Identification of a cleavage site directing the immunochemical detection of molecular abnormalities in type IIA von Willebrand factor. Proc Natl Acad Sci USA. 1990;87(16):6306-6310.

11. Sadler JE, Budde U, Eikenboom JC, et al. Working Party on von Willebrand Disease Classification. Update on the pathophysiology and classification of von Willebrand disease: a report of the Subcommittee on von Willebrand factor. J Thromb Haemost. 2006;4(10):2103-2114.

12. Goodeve A. The genetic basis of von Willebrand disease. Blood Rev. 2010; 24(3):123-134.

13. Stakiw J, Bowman M, Hegadorn C, et al. The effect of exercise on von Willebrand factor and ADAMTS-13 in individuals with type 1 and type 2B von Willebrand disease. J Thromb Haemost. 2008;6(1):90-96.

14. Pottinger BE, Read RC, Paleolog EM, et al. Von Willebrand factor is an acute phase reactant in man. Thromb Res. 1989;53(4):387-394.

15. Stirling Y, Woolf L, North WR, et al. Hemostasis in normal pregnancy. Thromb Haemost. 1984;52(2):176-182.

16. Daidone V, Galletta E, Casonato A. Type 1 von Willebrand disease due to a vicinal cysteine loss (p.C524Y) disclosed after a thrombotic episode. Thromb Res. 2018:161:91-93.

17. Daidone V, Gallinaro L, Cattini MG, et al. An apparently silent nucleotide substitution (c.7056C > T) in the von Willebrand factor gene is responsible for type 1 von Willebrand disease. Haematologica. 2011:96(6):881-887.

18. Pagliari MT, Baronciani L, Garcìa Oya I, et al. A synonymous (c.3390 C $>$ T) or a splicesite (c.3380-2A>G) mutation causes exon 26 skipping in four patients with von Willebrand disease (2A/IIE). J Thromb Haemost. 2013;11(7):1251-1259.

19. Corrales I, Ramírez L, Altisent C, Parra R, Vidal F. The study of the effect of splicing mutations in von Willebrand factor using RNA isolated from patients' platelets and leukocytes. J Thromb Haemost. 2011:9(4):679-688

20. Borràs N, Orriols G, Batlle J, et al. Unraveling the effect of silent, intronic and missense mutations on VWF splicing: contribution of next generation sequencing in the study of mRNA. Haematologica.
2019;104(3):587-598

21. Rodeghiero F, Tosetto A, Abshire T, et al on behalf of the ISTH/SSC Joint VWF and Perinatal/Pediatric Hemostasis Subcommittees Working Group. ISTH/SSC Bleeding Assessment Tool: a standardized questionnaire and a proposal for a new bleeding score for inherited bleeding disorders. J Thromb Haemost. 2010;8(9):20632065.

22. Casonato A, Pontara E, Sartorello F, et al. Reduced von Willebrand factor survival in type Vicenza von Willebrand disease. Blood. 2002;99(1):180-184

23. Casonato A, Pontara E, Sartorello F, et al. Identifying carriers of type $2 \mathrm{~N}$ von Willebrand disease: procedures and significance. Clin Appl Thromb Hemost. 2007:13(2):194-200.

24. Casonato A, Pontara E, Sartorello F, et al. Identifying type Vicenza von Willebrand disease. J Lab Clin Med. 2006;147(2):96102.

25. Casonato A, Pontara E, Boscaro M, et al. Abnormalities of von Willebrand factor are also part of the prothrombotic state of Cushing's syndrome. Blood Coagul Fibrinolysis. 1999;10(3):145-151.

26. Gallinaro L, Cattini MG, Sztukowska M et al. A shorter von Willebrand factor survival in $\mathrm{O}$ blood group subjects explains how $\mathrm{ABO}$ determinants influence plasma von Willebrand factor. Blood. 2008; 111(7):3540-3545.

27. Casonato A, Pontara E, Doria A, et al. Lack of multimer organization of von Willebrand factor in an acquired von Willebrand syndrome. Br J Haematol. 2002; 116(4):899-904

28. Casonato A, Pontara E, Doria A, et al. An Arg760Cys mutation in the consensus sequence of the von Willebrand factor propeptide cleavage site is responsible for a new von Willebrand disease variant. Blood. 2003;101(1):151-156.

29. Baronciani L, Cozzi G, Canciani MT, et al Molecular defects in type 3 von Willebrand disease: updated results from 40 multiethnic patients. Blood Cells Mol Dis. 2003; 30(3):264-270

30. Rehemtulla A, Kaufman RJ. Preferred sequence requirements for cleavage of provon Willebrand factor by propeptide-processing enzymes. Blood. 1992;79(9):2349-2355.

31. Platè $M$, Duga $S$, Baronciani $L$, et al Premature termination codon mutations in the von Willebrand factor gene are associated with allele-specific and position-dependent mRNA decay. Haematologica. 2010; 95(1):172-174.

32. Casonato A, Galletta E, Sarolo L, Daidone V. Type $2 \mathrm{~N}$ von Willebrand disease: Characterization and diagnostic difficul- ties. Haemophilia. 2018:24(1):134-140.

33. Casonato A, Pontara E, Battiston M, et al. C2362F mutation gives rise to an ADAMTS13-resistant von Willebrand factor. Thromb Haemost. 2013;109(6):9991006.

34. Daidone V, Saga G, Barbon G, et al. The p.R1819_C1948delinsS mutation makes von Willebrand factor ADAMTS13-resistant and reduces its collagen-binding capacity. Br J Haematol. 2015;170(4):564-573.

35. Tsai HM, Nagel RL, Hatcher VB, Sussman II. Multimeric composition of endothelial cell-derived von Willebrand factor. Blood. 1989;73(8):2074-2076.

36. Dong JF, Moake JL, Nolasco L, et al. ADAMTS-13 rapidly cleaves newly secreted ultralarge von Willebrand factor multimers on the endothelial surface under flowing conditions. Blood. 2002;100(12):40334039.

37. De Ceunynck K, De Meyer SF Vanhoorelbeke K. Unwinding the von Willebrand factor strings puzzle. Blood. 2013;121(2):270-277

38. Crawley JT, de Groot R, Xiang Y, Luken $\mathrm{BM}$, Lane DA. Unraveling the scissile bond how ADAMTS13 recognizes and cleaves von Willebrand factor. Blood. 2011; 118(12):3212-3221.

39. Turner NA, Nolasco L, Ruggeri ZM, Moake JL. Endothelial cell ADAMTS-13 and VWF: production, release, and VWF string cleavage. Blood. 2009:114(24):5102-5111.

40. Michaux G, Pullen TJ, Haberichter SL, Cutler DF. P-selectin binds to the D'-D3 domains of von Willebrand factor in Weibel-Palade bodies. Blood. 2006; 107(10):3922-3924.

41. Solimando M, Baronciani L, La Marca S, et al. Molecular characterization, recombinant protein expression, and mRNA analysis of type 3 von Willebrand disease: Studies of an Italian cohort of 10 patients. Am J Hematol. 2012;87(9):870-874.

42. Galletta E, Daidone V, Zanon E, Casonato S. Type 3 von Willebrand disease mistaken for moderate haemophilia A: a lesson still to be learned. Haemophilia. 2018; 24(3):154-157.

43. Casonato A, Cattini MG, Barbon G Daidone V, Pontara E. Severe, recessive type 1 is a discrete form of von Willebrand disease: the lesson learned from the c.15343C $>$ A von Willebrand factor mutation. Thromb Res. 2015;136(3):682-686.

44. Burset M, Seledtsov IA, Solovyev VV. Analysis of canonical and non-canonical splice sites in mammalian genomes. Nucleic Acids Res. 2000;28(21):4364-4375.

45. Sibley CR, Blazquez L, Ule J. Lessons from non-canonical splicing. Nat Rev Genet. 2016;17(7):407-421 